\title{
CORRESPONDENCE
}

\section{Comment on: "Exploring the best treatment options for BRAF-mutant metastatic colon cancer"}

British Journal of Cancer (2020) 122:1724-1725; https://doi.org/ 10.1038/s41416-020-0819-5

An interesting and particularly welcome article by Taieb et al. ${ }^{1}$ describes treatment options for the management of bad-prognosis colorectal cancer (CRC) patients harbouring BRAF-mutated tumours. The current and future therapeutic strategies for this category of patients are based on well-established and complex preclinical data to which the authors paid insufficient attention.

One of the major advances in BRAF-mutated CRC is the setting of BRAFi-MEKi combinations. Of importance, although not discussed by the authors, this association of a BRAFi with a MEKi was dictated by the fact that BRAFi can induce neoplasia, most often cutaneous squamous cell carcinoma. ${ }^{2}$ This effect is attributed predominantly to paradoxical ERK activation (the ability of BRAFi to stimulate RAF signalling in BRAF wt condition, thus activating ERK and stimulating proliferation). ${ }^{3}$ Figure 1 of the paper ${ }^{1}$ makes no mention of this tissue-specific molecular aspect, which is important not only for mechanistic reasons, but is also of therapeutic interest since BRAFi, which evades paradoxical MAPK pathway activation, is currently in clinical development. ${ }^{4}$ Moreover, a definition of a paradox index has been set for BRAFi (vemurafenib, dabrafenib and encorafenib, PLX8394) as a means of quantifying a therapeutic window of high clinical efficacy (at least in BRAF-mutant melanoma cells) with minimal paradoxical ERK activation. ${ }^{5}$

The authors did mention another level of the underlying molecular complexity, i.e. the interruption by MEKi of negative feedback of ERK signalling at the initiation steps of MAPKinase signalling. ${ }^{6}$ This explains why, in the case of colorectal cancer cells and not melanoma cells (deprived of EGFR signalling), the addition of EGFRi is a necessary complement to the BRAFi-MEKi association. When advocating EGFRi in CCR, and more broadly, the use of monoclonal antibodies, the respective order of application of cetuximab versus bevacizumab is still a matter of debate. Cetuximab is an $\lg G 1$ and this characteristic confers to the drug the capacity to develop the clinically important mechanism of antibody-dependent cellular cytotoxicity (ADCC). ADCC was recently highlighted by others and us for its significant contribution to the global action mechanism of cetuximab.



Fig. 1 BRAF - mutated metastatic CRC: molecular bases for an optimal combination BRAFi - MEKi - cetuximab - bevacizumab - CPI. 
Of importance is the immune modulation generated by $\mathrm{ADCC}^{7}$ In brief, based on preclinical and clinical observations, cetuximabmediated ADCC, through natural killer-cell release of INFa, results in priming of cytotoxic T cells. ${ }^{7}$ Recent experimental and clinical data have revealed that bevacizumab, through its interaction with VEGF, may in fact restore normal endothelial cell diapedesis. This results in favourable tissular diffusion of cytotoxic $T$ lymphocytes instead of regulatory $T$ cells, the diffusion of which is facilitated by the deleterious impact of VEGF on endothelial cells. ${ }^{8}$ Thus, under bevacizumab treatment, adequate tumoural tissue redistribution of beneficial antitumour CD8 T cells is achieved in place of detrimental regulatory $\mathrm{T}$ cells. This background may support recent clinical results regarding the optimal order for cetuximab (priming CD8 T cells) versus bevacizumab (allowing optimal access of CD8 T cells to the tumoural bed) in first-line metastatic $\mathrm{CRC}^{9}$ and strengthens combination strategies also including immunotherapy by checkpoint inhibitors in unstable microsatellite BRAF-mutated CRC patients. The fact that BRAF + MEK inhibition positively affects the tumour microenvironment and immune modulation is thus a strong argument in favour of adding immunotherapy in the context of the cetuximab-BRAFi-MEKi/ bevacizumab combination with attention being paid to an optimal sequencing. The included figure recapitulates the molecular mechanisms considered above and seeks to clarify the particularly complex background-sustaining treatment options for metastatic BRAF-mutated CRC.

\section{AUTHOR CONTRIBUTIONS}

G.M. and J.G. made substantial contributions to study conception and design. GM. and J.G. were involved in drafting the paper. All authors contributed to and approved all drafts.

\section{ADDITIONAL INFORMATION}

Ethics approval and consent to participate This project did not constitute research. Therefore, ethics committee approval was not required.

Consent to publish Not applicable.

Data availability Not applicable.

Competing interests The authors declare no competing interests.
Funding information None.

Note This work is published under the standard license to publish agreement. After 12 months the work will become freely available and the license terms will switch to a Creative Commons Attribution 4.0 International (CC BY 4.0).

Publisher's note Springer Nature remains neutral with regard to jurisdictional claims in published maps and institutional affiliations.

Gérard Milano ${ }^{1}$ and Jocelyn $\mathrm{Gal}^{2}$ ${ }^{1}$ Oncopharmacology Unit and UNS EA 7497, Centre Antoine Lacassagne, University Côte d'Azur, Nice F-06189, France and ${ }^{2}$ Epidemiology and Biostatistics Department, Centre Antoine Lacassagne, University Côte d'Azur, Nice F-06189, France Correspondence: Gérard Milano (gerard.milano@nice.unicancer.fr)

\section{REFERENCES}

1. Taieb, J., Lapeyre-Prost, A., Laurent Puig, P. \& Zaanan, A. Exploring the best treatment options for BRAF-mutant metastatic colon cancer. Br. J. Cancer 121, 434-42 (2019).

2. Poulikakos, P. I., Zhang, C., Bollag, G., Shokat, K. M. \& Rosen, N. RAF inhibitors transactivate RAF dimers and ERK signalling in cells with wild-type BRAF. Nature 464, 427-430 (2010).

3. Hatzivassiliou, G., Song, K., Yen, I., Brandhuber, B. J., Anderson, D. J., Alvarado, R. et al. RAF inhibitors prime wild-type RAF to activate the MAPK pathway and enhance growth. Nature 464, 431-435 (2010).

4. Zhang, C., Spevak, W., Zhang, Y., Burton, E. A., Ma, Y., Habets, G. et al. RAF inhibitors that evade paradoxical MAPK pathway activation. Nature 526, 583-586 (2015).

5. Adelmann, C. H., Ching, G., Du, L., Saporito, R. C., Bansal, V., Pence, L. J. et al. Comparative profiles of BRAF inhibitors: the paradox index as a predictor of clinical toxicity. Oncotarget 7, 30453-30460 (2016).

6. Corcoran, R. B., Ebi, H., Turke, A. B., Coffee, E. M., Nishino, M., Cogdill, A. P. et al. EGFR-mediated re-activation of MAPK signaling contributes to insensitivity of BRAF mutant colorectal cancers to RAF inhibition with vemurafenib. Cancer Discov. 2, 227-235 (2012).

7. Ferris, R. L., Lenz, H. J., Trotta, A. M., Garcia-Foncillas, J., Schulten, J., Audhuy, F. et al. Rationale for combination of therapeutic antibodies targeting tumor cells and immune checkpoint receptors: Harnessing innate and adaptive immunity through IgG1 isotype immune effector stimulation. Cancer Treat. Rev. 63, 48-60 (2018).

8. Munn, L. L. \& Jain, R. K. Vascular regulation of antitumor immunity. Science 365 544-545 (2019)

9. Bennouna, J., Hiret, S., Bertaut, A., Bouche, O., Deplanque, G., Borel, C. et al. Continuation of bevacizumab vs cetuximab plus chemotherapy after first progression in KRAS wild-type metastatic colorectal cancer: The UNICANCER PRODIGE18 Randomized Clinical Trial. JAMA Oncol. 5, 83-90 (2019). 